\title{
HPV: Unbefriedigender Impfstatus
}

Obwohl für Mädchen im Alter zwischen 12 und 17 Jahren empfohlen, wird in Deutschland weniger als die Hälfte dieser Mädchen gegen humane Papillomviren (HPV) geimpft. Diesen betrüblichen Umstand machte das Robert Koch-Institut (RKI) zusammen mit den Kassenärztlichen Vereinigungen (KVen) Anfang dieses Jahres im sogenannten Versorgungsatlas publik [Rieck T et al. HPV-Impfquoten im Regionalvergleich: Eine Sekundärdatenanalyse aus der KV-Impfsurveillance. RKI Fachgeb. 33 - Impfprävention]. Während bundesweit weniger als $1 \%$ der 12-Jährigen die drei erforderlichen Immunisierungen aufwiesen, zeigte sich bei 17-Jährigen eine Komplett-Impfquote von 40\%. Immerhin stiegen die Quoten in den Altersgruppen linear an. Für seine Untersuchung hat das Wissenschaftler-Team des RKI vertragsärztliche Abrechnungsdaten aus den Jahren 2008 bis 2013 von 16 der insgesamt 17 KVen ausgewertet. Das Forscherteam zog eine Stichprobe mit 1,13 Millionen Mädchen, die knapp die Hälfte der 12- bis 17-jährigen Mädchen im Jahre 2013 repräsentierte. Erstmals wurden Impfquoten nach Altersgruppe und bis auf Kreisebene ausgewertet.

Martin Roos

\section{Empfehlungen von Harald zur Hausen}

Der Beweis, dass humane Papillomviren (HPV) Gebärmutterhalskrebs auslösen können, hatte Harald zur Hausen 2008 den Nobelpreis eingetragen. Seine jahrzehntelange Forschung lieferte dann die Basis für eine Maßnahme zur Krebsprävention gleich mit: die HPV-Impfung. Diese würde gegenwärtig aber zu selten genutzt, so zur Hausen in einer Pressemitteilung des Deutschen Krebsforschungszentrums (DKFZ). Dabei ließe sich durch die Impfung ein nahezu 100\%iger Schutz gegen die häufigsten Erreger - HPV 16 und 18 - erzielen. Zugleich sei die Impfung sehr sicher. Zur Verbesserung der Impfraten empfiehlt zur Hausen kontinuierliche Aufklärung und schulische Impfprogramme. Er plädiert zudem dafür, auch Jungen zu impfen. Schließlich seien diese durch ihre im Vergleich zu den Mädchen größere Zahl an Sexualkontakten die wichtigste Verbreitungsquelle der Viren. Moritz Borchers

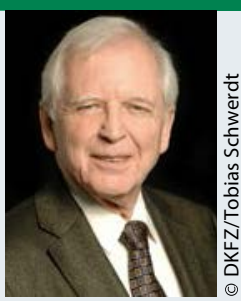

Harald zur Hausen, Nobelpreisträger für Medizin 2008

\section{USA vor ähnlichem Bild}

Am Fred-Hutchinson-Krebsforschungszentrum (FHKFZ) in Seattle legten Denise Galloway und Kollegen vor 25 Jahren die klinischen Grundsteine für die HPV-Vakzine. Nun brechen das FHKFZ und Galloway eine Lanze dafür, die US-Impfquote (unter $40 \%$ ) zu erhöhen, und schließen sich mit anderen US-Zentren zu einer Initiative zusammen: Im Rahmen des Regierungsprogramms "Healthy People 2020 " soll die Impfrate auf rund $80 \%$ angehoben werden.

Martin Roos 